\title{
Molecular mechanisms of decreased smooth muscle differentiation marker expression after vascular injury
}

\author{
Christopher P. Regan, ${ }^{1}$ Paul J. Adam, ${ }^{1}$ Cort S. Madsen, ${ }^{2}$ and Gary K. Owens ${ }^{1}$ \\ ${ }^{1}$ Department of Molecular Physiology and Biological Physics, University of Virginia, Charlottesville, Virginia, USA \\ ${ }^{2}$ Bristol Myers Squibb, Cardiovascular Drug Discovery, Princeton, New Jersey, USA \\ Address correspondence to: Gary K. Owens, Department of Molecular Physiology and Biological Physics, \\ 1300 Jefferson Park Avenue, PO Box 800736, Charlottesville, Virginia 22908, USA. \\ Phone: (804) 924-2652; Fax: (804) 982-0055; E-mail: gko@virginia.edu. \\ Christopher P. Regan's present address is: Vertex Pharmaceuticals, Department of Genomic Pharmacology, \\ Cambridge, Massachusetts, USA. \\ Paul J. Adam's present address is: Division of Cardiovascular Medicine, Department of Medicine, University of Cambridge, \\ Cambridge, United Kingdom. \\ Received for publication June 7, 2000, and accepted in revised form September 26, 2000.
}

\begin{abstract}
While it is well established that phenotypic modulation of vascular smooth muscle cells (VSMCs) contributes to the development and progression of vascular lesions, little is known regarding the molecular mechanisms of phenotypic modulation in vivo. Here we show that vascular injury reduces transcription of VSMC differentiation marker genes, and we identify cis regulatory elements that may mediate this decrease. Using a carotid wire-injury model in mice carrying transgenes for smooth muscle $\alpha$-actin, smooth muscle myosin heavy chain, or a SM $22 \alpha$ promoter- $\beta$-gal reporter, we collected arteries 7 and 14 days after injury and assessed changes in endogenous protein and mRNA levels and in $\beta$-gal activity. Endogenous levels for all markers were decreased 7 days after injury and returned to nearly control levels by 14 days. $\beta$-gal staining in all lines followed a similar pattern, suggesting that transcriptional downregulation contributed to the injury-induced decreases. To begin to dissect this response, we mutated a putative G/C-rich repressor in the $S M 22 \alpha$ promoter transgene and found that this mutation significantly attenuated injury-induced downregulation. Hence, transcriptional downregulation contributes to injury-induced decreases in VSMC differentiation markers, an effect that may be partially mediated through a G/C-rich repressor element.
\end{abstract}

J. Clin. Invest. 106:1139-1147 (2000).

\section{Introduction}

Percutaneous transluminal coronary angioplasty and other vascular reconstructive procedures (atherectomy, laser ablation, grafting, stenting) are effective means of revascularization of occluded arteries. However, because each procedure causes some extent of vascular trau$\mathrm{ma}$ /injury, the benefits are often limited by the risk of postprocedural restenosis. In fact, data from a variety of clinical studies indicate that the incidence of restenosis ranges from $25 \%$ to $50 \%$ for all interventional revascularization techniques (1-3).

Although it is clear that postprocedural restenosis involves a complex pathophysiological response, a key factor in the development of restenotic lesions is the phenotypic modulation of vascular smooth muscle cells (VSMCs). After vascular injury, VSMCs become phenotypically modulated and exhibit marked differences in morphology, migration, proliferation rate, and protein expression compared with normal medial VSMCs (4). One characteristic feature of phenotypically modulated VSMCs is that they express markedly lower levels of proteins (differentiation marker proteins) required for the VSMCs' normal, differentiated function, including smooth muscle $\alpha$-actin (SM $\alpha A)$, smooth muscle myosin beavy chain (SMMHC), and SM22 $\alpha$ (5-7).
Given that phenotypic modulation of VSMCs is a key factor in the development and progression of restenotic as well as atherosclerotic lesions, elucidation of the molecular mechanisms involved in the regulation of VSMC differentiation marker genes in vivo is likely to contribute to a better understanding of the disease process and to the development of novel therapeutic agents that inhibit lesion formation/progression.

The decrease in VSMC differentiation marker proteins in intimal VSMCs is a common characteristic of all animal models of vascular injury/restenosis and atherosclerotic lesions $(5,6)$. In fact, investigators have shown both decreased protein and steady-state mRNA levels for VSMC differentiation markers in the developing intima in a variety of animal models of vascular injury/restenosis and in human atherosclerotic lesions $(4,7)$. Although these studies have shown a decrease in steady-state mRNA levels of a number of VSMC differentiation markers in the developing lesion, it is unclear whether these decreases were transcriptionally mediated or were due to destabilization of transcripts encoding for these markers. This distinction is important, as a factor known to be present in high levels at the site of vascular injury (8), PDGFBB, can induce rapid and selective destabilization of $\mathrm{mRNAs}$ encoding for VSMC 
Table 1

Promoter fragments used and overview of expression pattern observed in SM $\alpha A$-LacZ, SMMHC-LacZ, and SM22 $\alpha$-LacZ transgenic mice

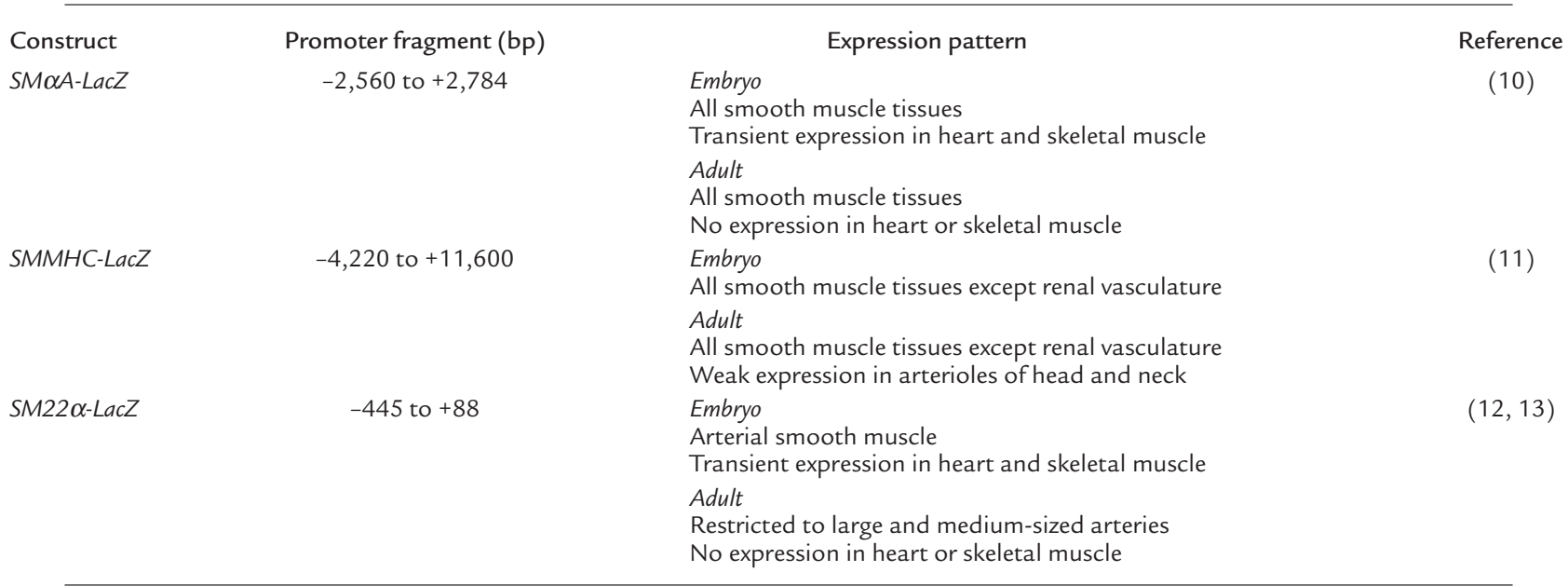

differentiation marker proteins (9). Although it has often been presumed that decreases in VSMC differentiation markers in phenotypically modulated VSMCs in vivo is mediated transcriptionally, there is no direct evidence to support this conclusion, and given previous in vitro studies, changes may be mediated solely by growth factor-induced posttranscriptional modifications.

To date, it has not been feasible to measure directly the transcriptional rate of VSMC differentiation marker genes in vivo after vascular injury. Recently, our laboratory has identified sufficient regions of both the $S M \alpha A$ (10) and SMMHC (11) promoters to drive expression of $\beta$-galactosidase $(\beta$-gal) in a manner that parallels the expression of the endogenous genes throughout embryonic development and in adult mice (SM $\alpha A-L a c Z$ and SMMHC-LacZ, respectively). Furthermore, sufficient regions of the $S M 22 \alpha$ promoter were previously identified that drive expression of $\beta$-gal specifically in arterial smooth muscle in adult mice (SM22 $\alpha$-LacZ) $(12,13)$. The availability of these mice thus permit, for the first time to our knowledge, a means to assess directly changes in promoter activity after vascular injury, and to identify molecular mechanisms responsible for alterations in expression of these marker genes in vivo. As such, the current study addressed whether transcriptional downregulation was one mechanism that contributed to the decrease in levels of mRNA and protein of multiple VSMC differentiation markers after vascular injury.

Although it seems likely that changes in mRNA and protein levels of VSMC differentiation markers may follow a similar pattern as other models of vascular injury, the relative size of the mouse carotid and nature of injury is different. Therefore, we first characterized changes in endogenous mRNA and protein levels of multiple VSMC differentiation markers at various time points after injury. To determine whether vascular injury is associated with transcriptional downregulation of VSMC differentiation markers, we used multiple inde- pendent $S M \alpha A-L a c Z, S M M H C-L a c Z$, and $S M 22 \alpha-L a c Z$ transgenic founder lines and examined changes in $\beta$-gal expression as an assay of transcriptional activity of the promoter at various times after injury. We found a rapid decrease in both immunohistochemical staining and endogenous mRNA levels of $S M \alpha A, S M M H C$, and $S M 22 \alpha$ after vascular injury. There was also a significant decrease in $\beta$-gal staining in all transgenic lines after injury, consistent with a rapid decrease in the transcription of $S M \alpha A, S M M H C$, and $S M 22 \alpha$. As a first step in examining key cis regulatory elements that may mediate the transcriptional downregulation, we mutated a G/C-rich element in the SM22 $\alpha$ promoter, which based on in vitro studies of the SMMHC promoter, may function as a repressor (14). We found that the mutation did not affect the expression pattern of the transgene throughout development, but it significantly attenuated the decrease in promoter activity after vascular injury. Our results provide evidence that the decrease in VSMC differentiation markers in the developing intima is due to coordinate downregulation of the genes that encode for these markers. However, our study does not exclude the possibility that mechanisms in addition to transcriptional downregulation, such as changes in the mRNA stability of the endogenous VSMC differentiation marker transcripts, may also play a role. These studies also provide the first evidence to our knowledge of a negative cis regulatory element important in the downregulation of at least one VSMC differentiation marker gene after injury in vivo.

\section{Methods}

Generation of transgenic mice. Animal protocols were approved by The University of Virginia Animal Care and Use Committee. Transgenic founder lines were generated by standard microinjection techniques in collaboration with the University of Virginia transgenic mouse core facility (15). The DNA constructs used to generate stable founder lines are detailed in Table 1. 
Founder lines were then bred to produce offspring for use in determining both basal $\beta$-gal expression $(10,11)$ and changes in expression of $\beta$-gal after vascular injury.

Vascular injury. Vascular injury was performed on animals from at least two independent founder lines for each construct to rule out transgene insertional effects. Vascular injury was performed on 7- to 9-week-old male transgenics as described elsewhere (16). Sham-operated control mice from each transgenic line, in which the left external carotid was ligated, were also examined to ensure that the change in blood flow caused by the ligation of the external carotid did not cause a significant change in $\beta$-gal expression.

$\beta$-gal staining. At 7 or 14 days after injury, mice were anesthetized with pentobarbital $(100 \mathrm{mg} / \mathrm{kg})$ and perfusion fixed via the left ventricle with $2 \%$ formaldehyde $/ 0.2 \%$ glutaraldehyde, and both carotids were harvested. Carotids were then rinsed and stained for $\beta$-gal activity with a solution containing $5 \mathrm{mM} \mathrm{K} \mathrm{K}_{3} \mathrm{Fe}(\mathrm{CN})_{6}, 5 \mathrm{mM}$ $\mathrm{K}_{4} \mathrm{Fe}(\mathrm{CN})_{6}, 0.5 \mathrm{mg} / \mathrm{ml}$ bluo-gal (Life Technologies Inc., Grand Island, New York, USA), 0.01\% Na-deoxycholate, and $0.2 \%$ NP-40. Care was taken to ensure that all samples were stained under the same conditions. Carotids were then processed for routine histology, and sections were counterstained with eosin. Carotid arteries that were used to determine lesion morphology, protein expression via immunohistochemistry, or steady-state mRNA levels via in situ hybridization were perfusion fixed with $10 \%$ formalin, harvested, immediately processed for paraffin embedding, and sectioned at $4 \mu \mathrm{m}$.

Western blot analysis. To determine the specificity of the SMMHC antibody, protein extracts were collected from rat and mouse aorta, cultured rat VSMCs, and cultured rat endothelial cells. Extracts were resolved via SDS/PAGE on a $4.5 \%$ acrylamide gel and transferred to a PVDF membrane. Blots were incubated in blocking solution $(50 \mathrm{mM}$ Tris, $150 \mathrm{mM} \mathrm{NaCl}, 5 \%$ milk, $0.1 \%$ Tween20) at room temperature for 1 hour. Blots were then incubated with rabbit anti-chicken SMMHC antibody (1:50,000; a gift from U. Groschel-Stewart; ref. 17), washed, and incubated with horseradish peroxidase-conjugated anti-rabbit $\operatorname{IgG}(1: 5,000)$ for 1 hour at room temperature. Blots were washed, and bound antibody was detected by enhanced chemiluminescence.

Immunohistochemistry. Immunohistochemical staining was performed using Vectastain ABC kit (Vector Laboratories, Burlingame, California, USA). Sections were deparaffinized and rehydrated, and endogenous peroxidases were quenched in $\mathrm{H}_{2} \mathrm{O}_{2}$ /methanol. After washing, sections were blocked with normal goat serum (for anti-SMMHC) or normal horse serum (for anti-SM $\alpha A$ ).
Anti-SM $\alpha A$ (clone 1A4; Sigma Chemical Co., St. Louis, Missouri, USA) was used at a dilution of 1:2,000. Anti$S M M H C$ was used at 1:1,000. The SMMHC antibody used in these studies has been shown to exhibit selective staining of SMC tissues in chicken (11). However, to our knowledge, this antibody has not been analyzed with respect to its reactivity and specificity in rodent tissue. This assessment is particularly important because considerable species variability in reactivity to nonmuscle myosin heavy chain variants has been observed with many SMMHC antibodies (4). We performed Western blot analysis on rat and mouse tissues as well as cultured cells. As seen in Figure 1, this antibody was specific for SM1/SM2 isoforms of SMMHC in rat and mouse carotid extracts (lanes 1 and 2), and it did not react with nonmuscle isoforms of myosin (lane 4). All sections were incubated for 1 hour at room temperature. Slides were then sequentially incubated with the appropriate biotinylated secondary antibody, peroxidase conjugated avidin-biotin complex, and enzyme visualization was accomplished with diaminobenzidine.

In situ hybridization. In situ hybridization was performed on paraffin-embedded sections as described previously (18) with minor modifications. Briefly, sections were rehydrated, blocked in acetic anhydride/triethanolamine, and digested with proteinase K. Sections were then washed, dehydrated, prehybridized for 1 hour at $50^{\circ} \mathrm{C}$, and hybridized with ${ }^{35} \mathrm{~S}$-UTP labeled antisense riboprobes specific for $S M \alpha A$ (19), SMMHC (SM1/SM2) (20), or $S M 22 \alpha$ (no. 631) overnight at $50^{\circ} \mathrm{C}$. Serial sections were hybridized with the corresponding sense riboprobes and served as controls. After hybridization, slides were incubated with RNase $A$ at room temperature and then washed in $1 \mathrm{xSSC} / 0.1 \% \mathrm{SDS}$ at $55^{\circ} \mathrm{C}$. Slides were then dipped in photographic emulsion (Kodak NTB2; Eastman Kodak Co., Rochester, New York, USA), exposed for 3 weeks, developed, and counterstained.

Nuclear extracts and electrophoretic mobility shift assay. Nuclear extracts were prepared from cultured VSMCs (21) according to the method of Dignam et al. (22). Electrophoretic mobility shift assays (EMSAs) were performed as described previously (23) with the following oligonucleotides (Operon Technologies Inc., Alameda, California, USA): MHCgc, 5'-ggttgtttccegcccaggcc-3'; SM22gc, 5'-ttttcceggecgecctcagc-3'; SM22gc $\Delta, \quad 5^{\prime}$ ttttcatccctcagc-3' which were ${ }^{32}$ P-end-labeled. Antibodies to SP1 and SP3 were purchased from Santa Cruz Biotechnology Inc. (Santa Cruz, California, USA). Antibodies were added after the initial incubation of nuclear extract with probe and the reaction was incubated for an additional 15 minutes before electrophoresis.

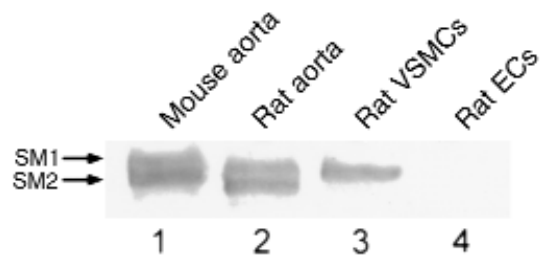

Figure 1

Western blot analysis of the SMMHC antibody used to assess changes in SMMHC protein levels after injury. Protein extracts from rat and mouse aorta, cultured VSMCs, and cultured endothelial cells were separated by SDS-PAGE, immobilized on a PVDF membrane and probed with a rabbit anti-chicken SMMHC antibody (17) as described in Methods. SM1 and/or SM2 isoforms of $S M M H C$ were detected in protein extracts from mouse aorta (lane 1), rat aorta (lane 2), and cultured rat VSMCs (lane 3 ), but not cultured rat endothelial cells (lane 4). 
Figure 2

Histological assessment of vessel morphology and immunohistochemical analysis of $S M \alpha A$ and $S M M H C$ protein of mouse carotid arteries post injury. Mice were sacrificed 7 days or 14 days after injury, and carotids were collected and fixed in 10\% formalin (see Methods). Tissue was processed for routine histology and hemotoxylin and eosin-stained (a-d). Sections were also stained using standard immunohistochemical techniques with antibodies specific to $S M \alpha A$ (mouse antihuman, Sigma Chemical Co.) (e-h) or SMMHC (a gift from U. Groschel-Stewart) (17) (i-I). Visualization was accomplished by diaminobenzidine. Arrows denote the internal elastic lamina, and the arrowheads denote the external elastic lamina.

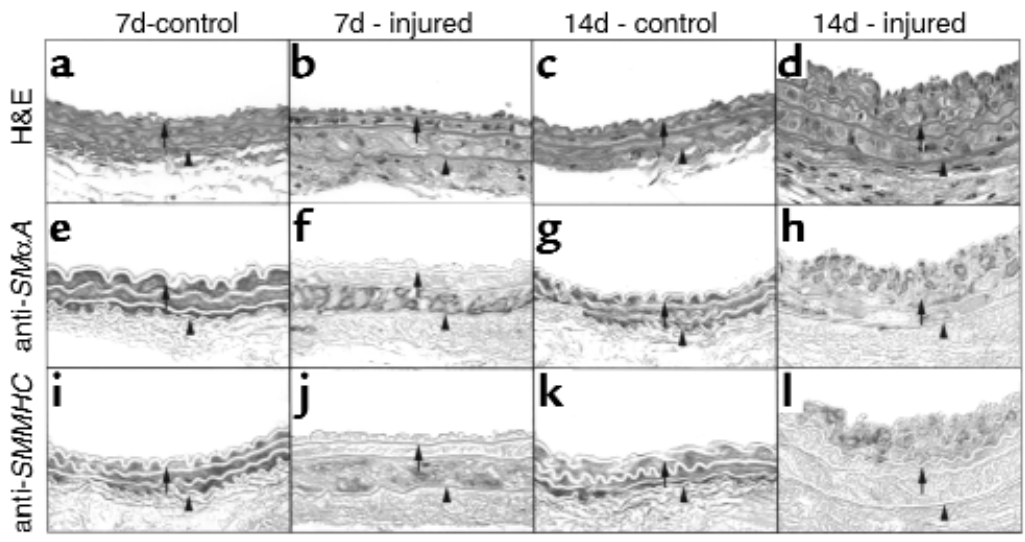

\section{Results}

Expression of the endogenous VSMC differentiation marker genes was decreased after injury. As the effect of vascular injury on temporal and spatial changes in $S M \alpha A$, $S M M H C$, and SM22 $\alpha$ expression has not been previously determined in this mouse model, we first determined changes in $S M \alpha A, S M M H C$, and $S M 22 \alpha$ protein and mRNA expression at 7 and 14 days after injury. Injury of the mouse carotid resulted in marked phenotypic changes throughout the medial wall (Figure 2). In contrast, in the rat model of balloon injury, phenotypic changes in VSMCs and decreased expression of VSMC differentiation markers were noted only within the developing intima and the most proximal medial SMC layers (5). Results of the present studies showed that cells throughout the media were enlarged and nuclei irregularly oriented 7 days after injury compared with uninjured carotids (Figure 2, compare a with b), and that these morphological changes were accompanied by decreased staining for SM $\alpha A$ and SMMHC (Figure 2 , e and $f$, and Figure 2, $i$ and $j$, respectively). Furthermore, results of in situ hybridization studies (Figure 3) showed that steady-state mRNA levels for $S M \alpha A, S M M H C$, and SM22 $\alpha$ were decreased throughout the medial wall and developing intima (Figure 3, b, $\mathrm{f}$, and $\mathrm{j}$, respectively). By 14 days, intimal thickness was increased significantly and was approximately equal to that of the media. Staining for both $S M \alpha A$ and
SMMHC was qualitatively increased in the injured artery at 14 days compared with 7 (Figure 2, compare f with h, and Figure 2 compare $\mathrm{j}$ with 1 , respectively), but staining for both of these SMC differentiation markers seemed somewhat less than the uninjured artery (Figure 2, compare g with h for $S M \alpha A$; Figure 2, compare k with 1 for $S M M H C)$. Similarly, mRNA levels for $S M \alpha A$, $S M M H C$, and $S M 22 \alpha$ were qualitatively increased at 14 compared with 7 days after injury, but seemed slightly less than in control, uninjured arteries (Figure 3).

The activity of the SM $\alpha A-L a c Z$, SMMHC-LacZ, and SM22 $\alpha$ $L a c Z$ transgenes were decreased after injury. The decrease in endogenous mRNA and protein could be due to either mRNA destabilization or decreased gene transcription. To determine whether the decreased levels of SMC differentiation markers were due to decreased gene transcription, the effects of injury on $\beta$-gal activity were studied in SM $\alpha$ A-LacZ, SMMHC-LaCZ, and SM22 $\alpha$-LacZ transgenic mice. A fragment of the $S M 22 \alpha$ promoter (Table 1), similar to the region that was previously shown to drive expression of $\beta$-gal in arterial smooth muscle $(12,13)$, was used to generate multiple independent founder lines. While it seems clear that further regulatory elements are needed to direct expression in all smooth muscle tissues in the adult, this transgene has clearly been shown to contain sufficient promoter sequence for arterial expression $(12,13)$. The expression pattern of promoter fragment throughout development and in
Figure 3

In situ hybridization analyses of $S M \alpha A, S M M H C$, and SM22 $\alpha$ at 7 and 14 days after carotid injury. Mice were sacrificed 7 or 14 days after injury, and carotids were collected, fixed in $10 \%$ formalin, and processed for paraffin sectioning. Sections were treated as described in Methods, hybridized with ${ }^{35}$ S-UTP labeled antisense riboprobes specific for $S M \alpha A$ (a-d), SMMHC (e-h), or SM22 $\alpha(\mathbf{i}-\mathrm{I}) \mathrm{mRNA}$ for 16 hours at $50^{\circ} \mathrm{C}$, treated with RNaseA, and washed at $55^{\circ} \mathrm{C}$ in $1 \times \mathrm{SSC} / 0.1 \% \mathrm{SDS}$. Sections were then exposed to photographic emulsion for 3 weeks. Serial sections probed with sense riboprobes served as controls. There was little nonspecific binding of any sense probe (data not shown). Arrows denote the internal elastic lamina, and the arrowheads denote the external elastic lamina.
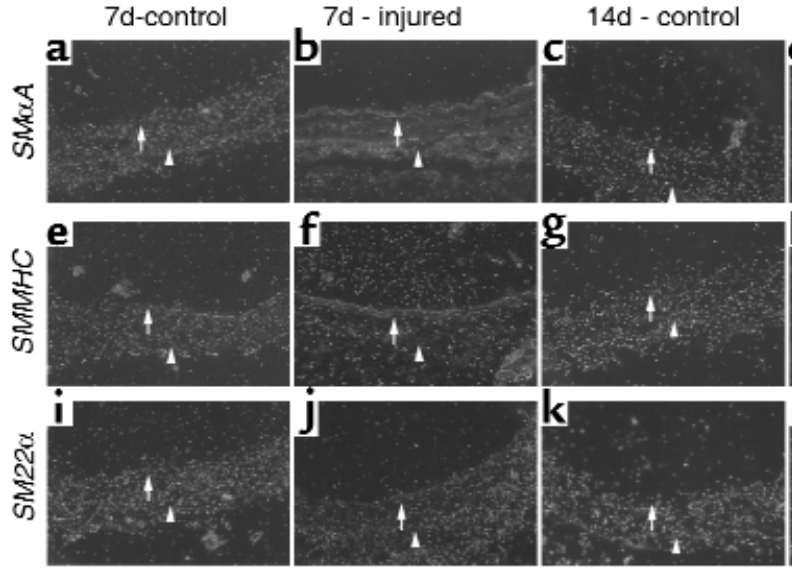

$14 \mathrm{~d}$ - injured

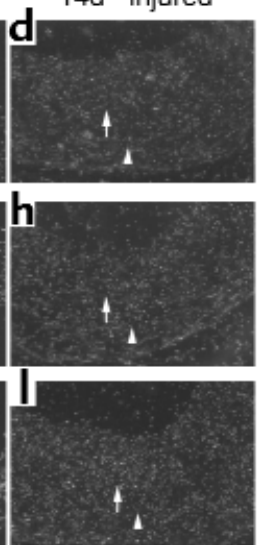


adult mice (Figure 5, top) used in the current study was identical to that reported previously $(12,13)$. We also used $S M \alpha A-L a c Z$ and SMMHC-LacZ transgenic mice (Table 1), developed previously in our laboratory $(10,11)$, that virtually recapitulate the expression of the endogenous genes throughout development and in adult mice. To determine whether vascular injury was associated with changes in promoter activity of multiple SMC differentiation markers in vivo, carotid injury was performed on at least two independent transgenic founder lines of SM $\alpha A-L a c Z, S M M H C-L a c Z$, and SM22 $\alpha-L a c Z$ transgenic mice. Carotids were harvested at 7 or 14 days after injury, stained for $\beta$-gal activity, and examined histologically to assay for changes in $\beta$-gal expression. A subset of mice from each line were subjected to external carotid occlusion without injury to ensure that the change in blood flow caused by ligation of the external carotid did not alter $\beta$-gal staining. These mice showed normal staining of both carotids (data not shown), similar to that seen in the contralateral control artery.

Overall, $\beta$-gal staining agreed closely with immunostaining and in situ. However, it is interesting to note that at certain times, $\beta$-gal staining seemed somewhat less than immunostaining or steady-state mRNA levels. For example, immunostaining and in situ hybridization signal for endogenous SMMHC seem slightly greater than $\beta$-gal staining at the same time point (compare Figure 2, part 1; Figure 3h; and Figure 4h). These small discrepancies are not surprising given the difficulties in directly comparing results across assays of this type due to variations in assay sensitivity, half-life of product being detected, etc.; therefore, comparisons across assays of this type must be interpreted cautiously. In $S M \alpha A$-LacZ mice, $\beta$-gal was significantly decreased in the injured vessel in both the media and developing intima 7 days after injury compared with control (Figure 4, compare b with a), suggesting that $S M \alpha A$ transcription was rapidly downregulated. At 14 days after injury, $\beta$-gal activity was increased in the injured artery compared with that at 7 days (Figure 4, compare d with b). However, the expression was still less than in the media of the uninjured carotid (Figure 4, compare d with c). There was a marked decrease in transcription of the SMMHC-LacZ transgene at 7 days after injury, in the media and intima compared with control (Figure 4, compare b with a). Fourteen days after injury, $\beta$-gal expression in SMMHC-LacZ mice was increased in the media and intima compared with that at 7 days (Figure 4, compare h with $\mathrm{f}$ ), suggesting increased promoter activity. However, staining was still less than in the uninjured control (Figure 4, compare h with g). As observed in the $S M \alpha A$-LacZ and SMMHC-LacZ mice, $\beta$-gal expression in the entire media and developing intima was significantly decreased 7 days after injury in SM22 $\alpha$-LacZ mice (Figure 4, compare $\mathrm{j}$ with $\mathrm{i}$ ). $\beta$-Galactosidase expression was increased significantly in the media and

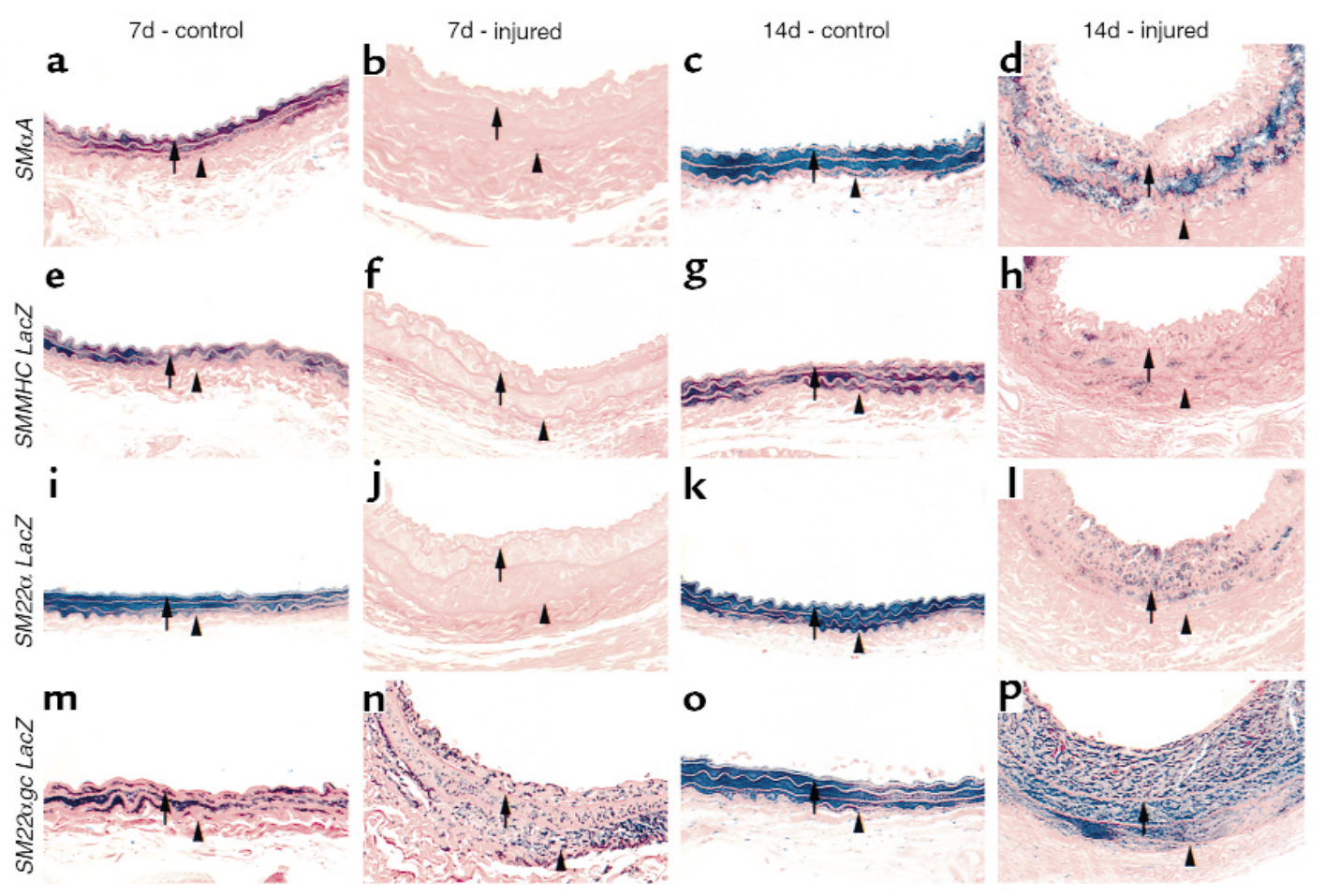

Figure 4

$\beta$-Galactosidase staining of mouse carotid arteries from SM $\alpha A$ (a-d), SMMHC-LacZ (e-h), SM22 $\alpha$-LacZ (i-I), and SM22 $\alpha g c-L a c Z$ (m-p) transgenic mice 7 or 14 days after injury or in uninjured controls. The left common carotid was injured as described in Methods, and the right carotid served as control. Carotids were harvested, stained for $\beta$-gal activity, processed for histology, and counterstained with eosin. Arrows denote the internal elastic lamina, and the arrowheads denote the external elastic lamina. 
intima of SM22 $\alpha$-LacZ mice 14 days after injury, but the expression still seemed slightly lower than in uninjured control arteries (Figure 4, compare 1 with $\mathrm{k}$ ). Taken together, these results indicate that vascular injury is associated with a rapid transcriptional downregulation of $S M \alpha A, S M M H C$, and SM22 $\alpha$. Moreover, the data suggest possible coordinate downregulation of multiple SMC differentiation markers after vascular injury.

Mutation of an SM22 $\alpha \mathrm{G} / \mathrm{C}$-rich repressor element attenuated the injury-induced decrease in transgene expression. As a first step to discerning molecular mechanisms that might contribute to transcriptional repression of VSMC differentiation markers after injury, sequence comparisons were made among the $S M \alpha A, S M M H C$, and $S M 22 \alpha$ transgene promoters to identify potential common cis elements that might contribute to the transcriptional repression of these promoters. Although no conserved candidate repressor element was found to be present in all three promoters, the SM22 $\alpha$ and SMMHC promoters share a conserved $\mathrm{G} / \mathrm{C}$-rich element located proximal to the distal CArG element. Previous studies of the SMMHC promoter showed that its G/C-rich element bound SP1/SP3 and that mutation of this element resulted in dramatically increased promoter activity in transfected cultured VSMCs (14). To determine the role of this G/C-rich element on SM22 $\alpha$ transgene expression in vivo, transgenic mice in which this $\mathrm{G} / \mathrm{C}$ element was mutated (SM22 $\alpha g c-L a c Z$ ) were generated, and transgene expression was examined throughout development and after vascular injury. Mutation of the $\mathrm{G} / \mathrm{C}$ repressor had no effect on $\beta$-gal expression throughout development or in adult tissue (Figure 5). However, this mutation dramatically attenuated the decrease in the SM22 $\alpha$-LacZ transgene activity after injury (Figure 4, m-p). For example, expression of the SM22 $\alpha$ LacZ transgene was nearly abolished 7 days after injury. By 14 days, $S M 22 \alpha$-LacZ transgene activity was significantly increased compared with that at 7 days, but still slightly lower than uninjured control arteries (Figure 4, i-l). However, mutation of the $\mathrm{G} / \mathrm{C}$-rich region significantly attenuated this decrease after injury (Figure 4, m-p). Although $\beta$-gal expression 7 days after injury in

\section{Figure 5}

Basal expression of SM22 $\alpha$-lacZ and SM22 $\alpha g c$ $L a c Z$ transgenes at various embryonic time points and in adult aorta. Embryos and adult tissues were collected, stained for LacZ activity, and cleared as described previously (11). There was no qualitative differences in expression pattern due to the mutation of the $\mathrm{G} / \mathrm{C}$-rich region.
$S M 22 \alpha g c-L a c Z$ mice was lower than in uninjured control arteries (Figure 4, compare $n$ with $\mathrm{m}$ ), the $\beta$-gal expression in injured arteries from SM22 $\alpha$ gc-LacZ mice was significantly higher than injured arteries from SM22 $\alpha$-LacZ (Figure 4, compare $n$ with j). Similarly, 14 days after injury, $\beta$-gal expression in injured arteries from $S M 22 \alpha g c$ $L a c Z$ mice was still qualitatively higher than that of $S M 22 \alpha$-LacZ mice (Figure 4, compare $\mathrm{p}$ with $\mathrm{l}$ ) and similar to that in uninjured control arteries (Figure, compare $p$ with o). These data suggest that the G/C-rich element plays a critical role in the transcriptional regulation of the SM22 $\alpha$ promoter after vascular injury in vivo.

Previous studies in our laboratory showed that the SMMHC G/C repressor element bound Sp1/Sp3 and that $\mathrm{Sp} 1 / \mathrm{Sp} 3$ expression was increased within vascular lesions (14). To determine whether the SM22 $\alpha \mathrm{G} / \mathrm{C}$ region also bound $\mathrm{Sp} 1 / \mathrm{Sp} 3$, EMSAs were performed with a 20-bp oligonucleotide probe containing the SM22 $\alpha \mathrm{G} / \mathrm{C}$ element plus $5^{\prime}$ and $3^{\prime}$ flanking sequence. As a control, we also examined binding to a 20-bp probe containing the SMMHC G/C element and flanking sequence. Results showed that incubation of either the SMMHC (lane 1) or SM22 $\alpha$ (lane 6) G/C-rich-containing probes with SMC nuclear extract resulted in the formation of two distinct shift bands (Figure 6, arrows). Both the lower and upper mobility shift bands could be supershifted with antibodies to Sp3 (Figure 6, lanes 2, 4, and 5) or Sp1 (Figure 6, lanes 7, 9, and 10), respectively. Moreover, both the SMMHC and $S M 22 \alpha$ $\mathrm{G} / \mathrm{C}$ element containing oligonucleotide probes bound recombinant SP1 (Figure 6, lanes 3 and 8). Importantly, mutation of the $S M 22 \alpha \mathrm{G} / \mathrm{C}$-rich element in a man-
$11.5 d$
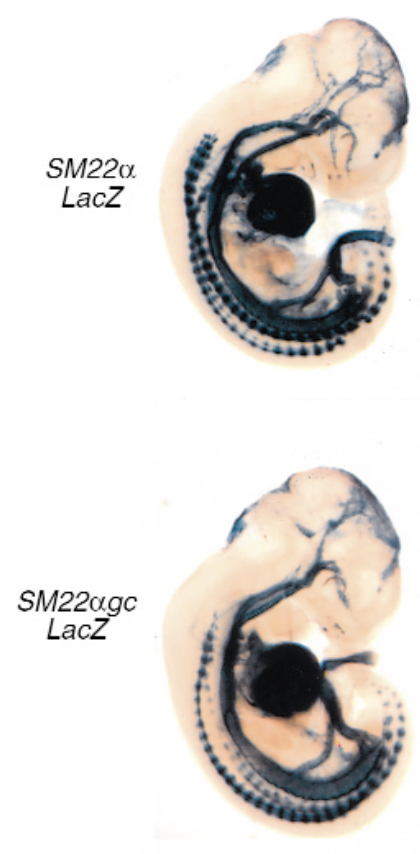

$3.5 d$
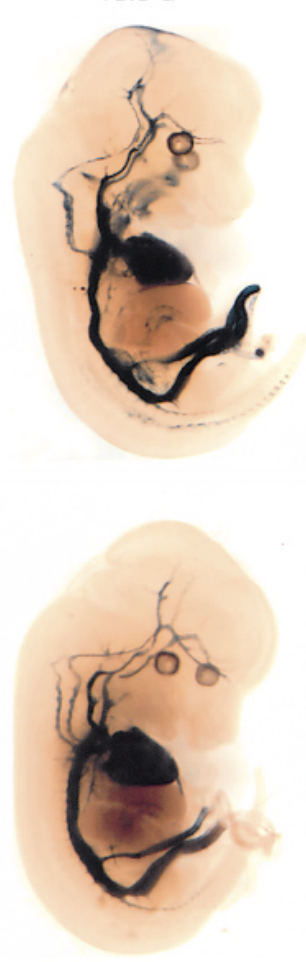
ner identical to that examined in transgenic studies (Figures 4 and 5), resulted in complete abolition of binding of Sp1 and Sp3 to this probe (Figure 6, lanes 11 and 12). Taken together, results provide clear evidence that the $S M 22 \alpha \mathrm{G} / \mathrm{C}$ repressor element is capable of binding the transcription factors Sp1 and Sp3.

\section{Discussion}

Although postprocedural restenosis and the development of atherosclerotic lesions are complex pathophysiological responses that involve many components, including activation of inflammatory pathways, adventitial remodeling, vessel remodeling, and intimal thickening (1, 24-26), a key factor in the development and progression of these lesions is the phenotypic modulation of VSMCs. The factors that initiate phenotypic modulation of VSMCs are not well understood. However, a critical step in the process is the concomitant decrease in a variety of VSMC differentiation markers, including $S M \alpha A, S M M H C$, and $S M 22 \alpha$ (4). Although much has been learned from examining VSMC gene regulation in cultured cells, in vitro conditions cannot fully recapitulate the variety of factors involved in the regulation of these genes after vascular injury in vivo $(4,11)$. Therefore, studies aimed at examining the regulation of these genes in vivo are critical to dissect the factors and molecular mechanisms that regulate phenotypic modulation of intimal VSMCs. Moreover, such studies may contribute to the development of novel therapies aimed at controlling VSMC phenotypic alterations.

The data presented in this report provide convincing evidence that vascular injury in the mouse, as in other species, induces marked decreases in expression of $S M \alpha A, S M M H C$, and $S M 22 \alpha$ at the protein and mRNA level. A major goal of the present studies was to determine whether transcriptional repression contributed to injury-induced decreases in expression of these genes. Therefore, we used multiple smooth muscle promoter LacZ transgenic lines as a means to assess the transcriptional activity of the endogenous genes encoding for these proteins. Results provide clear evidence showing that injury resulted in marked downregulation in expression of each of these smooth muscle promoter transgenes (Figure 4). Of key importance, we previously showed that the expression of the $S M \alpha A$ and $S M M H C$ transgenes used in the present studies closely mimics their endogenous genes throughout development and maturation $(10,11)$. Moreover, studies by others have shown that activity of the SM22 $\alpha$ transgene is sufficient to appropriately regulate this gene within arterial smooth muscle during development and maturation (12). Indeed, we selected multiple independent founder lines to perform the present studies in which the correlation between transgene and endogenous gene expression was extremely high. As such, we believe that assessments of transgene activity provide a valid estimate of activity of endogenous gene expression and that our results provide compelling evidence that reductions in transcription of $S M \alpha A, S M 22 \alpha$, and
SMMHC contributed to phenotypic modulation of smooth muscle cells in response to vascular injury. Nevertheless, it must be appreciated that because the insertional locus of transgenes differs from that of endogenous genes, our experimental approach may not fully recapitulate all regulatory processes normally involved in transcriptional regulation of these genes in response to injury including processes associated with control of chromatin structure (27).

Results of the present studies provide novel evidence indicating that injury-induced suppression of SM22 $\alpha$ gene expression was markedly attenuated by mutation of a $\mathrm{G} / \mathrm{C}$ repressor element located within the $5^{\prime}$ promoter region. This finding is of considerable interest because it provides a definitive model system with which to identify signaling mechanisms and extrinsic factors that contribute to phenotypic modulation of SMC in vivo. A critical first step will be to identify mechanisms and factors that regulate $S M 22 \alpha$ promoter activity through the G/C-rich region in vivo. There are several lines of evidence that suggest that transcriptional repression through the G/C-rich region may involve SP1/SP3.

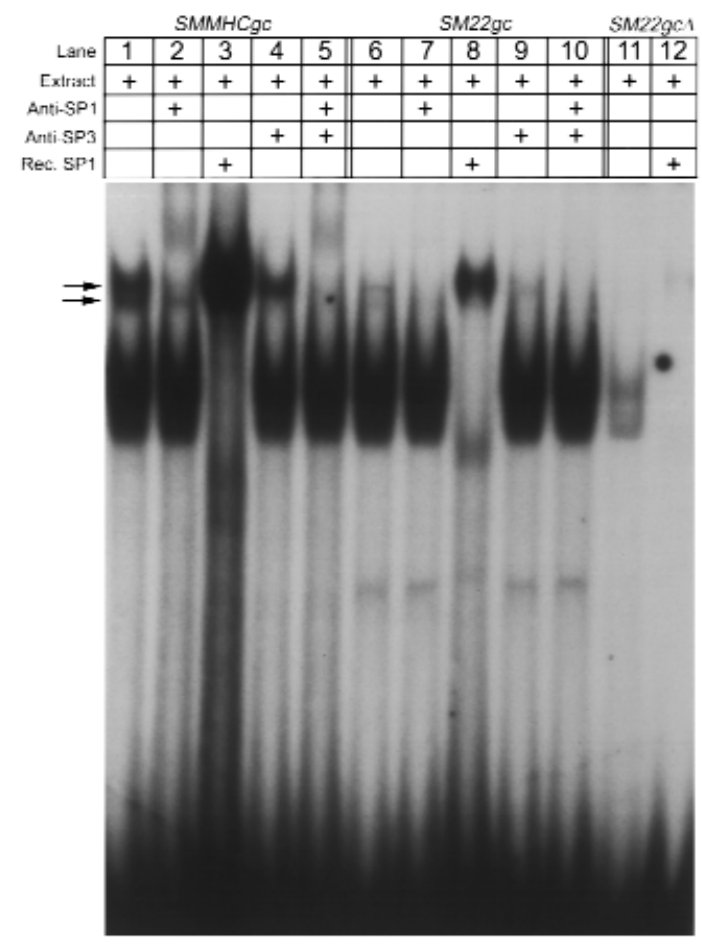

\section{Figure 6}

EMSA of SP $1 /$ SP 3 binding to the SMMHC and SM $22 \alpha \mathrm{G} / \mathrm{C}$-rich element and effect of deletion mutant in $S M 22 \alpha .{ }^{32} \mathrm{P}$ end-labeled oligonucleotides probes were as described in Methods. Incubation of radiolabeled probes with cultured VSMC nuclear extracts resulted in two distinct shift bands with either the SMMHC or SM22 $\alpha \mathrm{G} / \mathrm{C}$-rich elements (lanes 1 and 6). Antibodies to SP1 and SP3 supershifted the upper and lower bands, respectively (lanes 2, 4, and 5 and 7, 9, and 10, respectively). Human recombinant SP1 (Promega Corp., Madison, Wisconsin, USA) also bound both G/C-rich elements (lanes 3 and 8). The G/C deletion used to generate SM22gc-LacZ mice abrogated binding of SP1 and SP3 (lane 11) from nuclear extracts as well as recombinant SP1 (lane 12). Rec., recombinant. 
First, SP1/SP3 have been shown to be present in the developing intima in other models of vascular injury (14, 28). Second, we found that the $S M 22 \alpha \mathrm{G} / \mathrm{C}$-rich region bound SP1/SP3 in vitro (Figure 6). However, there is no direct evidence that SP1/SP3 contribute to the repressor effect in vivo, and one must also consider the possibility that other SP1-like transcription factors may be involved in the regulation of promoter activity in vivo. For example, there is evidence implicating a variety of zinc finger transcription factors in regulation of gene expression after vascular injury. SP1-like factors are primarily restricted to binding sequences that are rich in $\mathrm{G}$ or $\mathrm{C}$, but the sequence specificity is somewhat relaxed (29). Watanabe et al. (28) recently showed that expression of an SP1-like transcription factor, BTEB2, was upregulated in the developing intima in balloon-injured rat aorta. They also found that BTEB2 transactivated the nonmuscle myosin heavy chain B (NMMHC-B) promoter in transiently transfected cells, suggesting that its presence in the lesion may upregulate NMMHC-B. However, its role in regulating VSMC differentiation marker genes, in vitro or in vivo, has not been determined. Several SP1like transcription factors have also been show to mediate PDGFBB expression in cultured VSMCs (30), suggesting a role in growth factor expression and, possibly, VSMC phenotypic modulation after injury.

Although SP1 is generally thought to activate transcription, previous studies have demonstrated that it can function as a repressor as well. For example, Khachigian et al. (31) demonstrated that Egr-1 displaced SP1 from the PDGF-A chain promoter to activate transcription, suggesting a role for SP1 as a repressor. Madsen et al. (14) showed that SP1 bound to a G/C-rich repressor region in the $S M M H C$ promoter and mutation of this region significantly enhanced promoter activity in vitro. Additionally, they showed increased immunohistochemical staining for SP1 in the developing intima in injured rat carotid arteries suggesting an association between decreased levels of VSMC differentiation markers, such as SMMHC, and increased levels of SP1 (14). These latter observations are particularly interesting because the $\mathrm{G} / \mathrm{C}$-rich region examined in the SMMHC promoter (14) is nearly identical to the $S M 22 \alpha \mathrm{G} / \mathrm{C}$-rich region examined in our study, suggesting a role for SP1-like factors in repression of multiple VSMC differentiation marker genes after injury. SP1 and SP3 have been shown to dynamically regulate other promoters through binding to the same cis element. For example, SP1-mediated activation of the flk-1 promoter was attenuated by SP3, suggesting that the regulation of this promoter may depend on variations in the ratio of SP1/SP3 (29). In light of these data, it is tempting to speculate that SP1/SP3 positively and negatively regulate promoter activity of VSMC differentiation markers through alterations in the levels of these transcription factors in phenotypically modulated VSMCs. However, it is also possible that other G/C-rich binding factors that are underrepresented (or absent) in our cultured cells, may be upregulated in VSMCs after injury and contribute to transcriptional repression. Further studies will be needed to identify and characterize factors that bind to the G/C-rich element in vivo, and to determine their role in transcriptional repression of multiple VSMC differentiation. Importantly, it must be emphasized that although the results of the present study demonstrated that mutation of the $S M 22 \alpha$ G/C-rich region attenuated the decrease in promoter activity after injury, the effect was not complete. This is not unexpected and suggests that additional cis repressor elements identified within this region (13) may also play an important role in mediating alterations in promoter activity after injury. Finally, there may be mechanisms in addition to decrease in gene transcription that affect endogenous levels of VSMCs differentiation marker genes after injury, such as growth-factor induced mRNA destabilization (9).

Although it is still unclear what external factors may regulate expression of negatively acting trans factors, there are a number of external factors that have been shown to alter the expression of VSMC differentiation marker genes following vascular injury. The physical trauma associated with the nature of vascular injury is likely to effect VSMC phenotype through disruption of cell-cell and cell-matrix interactions (32). In fact, removal of the endothelial lining of the rat carotid in a manner that did not cause VSMC trauma resulted in a smaller intimal lesion than balloon injury (33). Additionally, the high concentration of growth factors that may be present at the site of injury, in part derived from platelets that adhere to the lesion (16), may be another mechanism through which VSMCs gene regulation is altered. Moreover, the mechanical trauma associated with the injury may induce a variety of growth factors to be released or synthesized by VSMCs (34). Previous studies in cultured VSMCs have shown that PDGFBB induced a selective destabilization of $S M \alpha A$ mRNA without a change in the transcriptional rate of the gene in cultured VSMCs (9). While studies such as these provide important information regarding role of growth factors in VSMC differentiation marker gene regulation, it is often difficult to assess the role these factors play in vivo due to culture-induced alterations in VSMC phenotype (4). Therefore, studies have also addressed the role of specific growth factors after vascular injury by using neutralizing antibodies. For example, neutralizing antibodies directed against PDGFBB or bFGF administered after balloon injury reduced intimal lesion size (35). Although studies such as these clearly showed a role for these growth factors in intimal formation, they did not address the specific role of these factors on VSMC gene transcriptional regulation in vivo. Using the model described in the current study to monitor promoter activity, future studies using this model in combination with specific growth factor inhibition could begin to dissect the roles of specific growth factors in the transcriptional regulation of VSMCs. Furthermore, studies designed to use SMC specific promoter-reporter transgenics in combination with other models of lesion development, such as atherosclerotic and vascular allo- 
graft models, will provide a key step in determining mechanisms of VSMC gene regulation in vivo.

In summary, keeping in mind the caveats of transgenic analysis, results of the present studies provide strong evidence indicating that transcriptional downregulation contributes to the decreases in levels of $S M \alpha A, S M M H C$, and $S M 22 \alpha$ in VSMCs after injury in vivo. Furthermore, studies are the first to identify a negative cis regulatory element that is required for transcriptional repression of a VSMC selective gene after injury in vivo. Overall, we believe that the data presented are an important step in dissecting mechanisms that contribute to alterations in the transcriptional regulation of VSMC genes in response to vascular injury. Further studies examining factors that bind to the $\mathrm{G} / \mathrm{C}$-rich repressor element and identification of mechanisms that regulate the expression and activity of these factors in the development of vascular pathologies may also novel insights regarding the molecular control of VSMC phenotypic modulation in vascular disease. Moreover, the transgenic mouse injury model system described should have considerable utility for identifying specific signaling pathways and local environmental cues that contribute to phenotypic modulation of VSMCs in a variety of vascular pathologies.

\section{Acknowledgments}

This work was supported by grants from the NIH (RO1HL57353 and RO1HL38854 to G.K. Owens) and the American Physiological Society Fellowship in Physiological Genomics (to C.P. Regan). The authors thank Brian Duling, Kathy Day (University of Virginia Cardiovascular Research Center), and Angela Miller (University of Virginia Histology Core Facility), for technical support and/or helpful discussions.

1. Schofer, J., Rau, T., Schluter, M., and Mathey, D.G. 1997. Short-term results and intermediate-term follow-up of laser wire recanalization of chronic coronary artery occlusions: a single-center experience. J. Am. Coll. Cardiol. 30:1722-1728.

2. Radke, P.W., et al. 1999. Mechanisms of acute lumen gain and recurrent restenosis after rotational atherectomy of diffuse in-stent restenosis: a quantitative angiographic and intravascular ultrasound study. J. Am. Coll. Cardiol. 34:33-39.

3. Kastrati, A., et al. 1999. Prognostic value of the modified American College of Cardiology/American Heart Association stenosis morphology classification for long-term angiographic and clinical outcome after coronary stent placement. Circulation. 100:1285-1290.

4. Owens, G.K. 1995. Regulation of differentiation of vascular smooth muscle cells. Physiol. Rev. 75:487-517.

5. Kocher, O., et al. 1991. Phenotypic features of smooth muscle cells during the evolution of experimental carotid artery intimal thickening. Biochemical and morphologic studies. Lab. Invest. 65:459-470.

6. Aikawa, M., et al. 1998. Lipid lowering promotes accumulation of mature smooth muscle cells expressing smooth muscle myosin heavy chain isoforms in rabbit atheroma. Circ. Res. 83:1015-1026.

7. Weissberg, P.L., Cary, N.R., and Shanahan, C.M. 1995. Gene expression and vascular smooth muscle cell phenotype. Blood Press. Suppl. 2:68-73.

8. Lindner, V., Giachelli, C.M., Schwartz, S.M., and Reidy, M.A. 1995. A subpopulation of smooth muscle cells in injured rat arteries expresses platelet-derived growth factor-B chain mRNA. Circ. Res. 76:951-957.

9. Corjay, M.H., Blank, R.S., and Owens, G.K. 1990. Platelet-derived growth factor-induced destabilization of smooth muscle alpha-actin mRNA. $J$. Cell. Physiol. 145:391-397.

10. Mack, C.P., and Owens, G.K. 1999. Regulation of smooth muscle alphaactin expression in vivo is dependent on CArG elements within the 5 and first intron promoter regions. Circ. Res. 84:852-861.

11. Madsen, C., et al. 1998. Smooth muscle-specific expression of the smooth muscle myosin heavy chain gene in transgenic mice requires $5^{\prime}$ flanking and first intronic DNA sequence. Circ. Res. 82:908-917.

12. Li, L., Miano, J.M., Mercer, B., and Olson, E.N. 1996. Expression of the SM22alpha promoter in transgenic mice provides evidence for distinct transcriptional regulatory programs in vascular and visceral smooth muscle cells. J. Cell Biol. 132:849-859.

13. Kim, S., Ip, H.S., Lu, M.M., Clendenin, C., and Parmacek, M.S. 1997. A serum response factor-dependent transcriptional regulatory program identifies distinct smooth muscle cell sublineages. Mol. Cell. Biol. 17:2266-2278.

14. Madsen, C., Regan, C., and Owens, G. 1997. Interaction of CArG elements and a GC-rich repressor element in transcriptional regulation of the smooth muscle myosin heavy chain gene in vascular smooth muscle cells. J. Biol. Chem. 272:29842-29851.

15. Gordon, J.W., and Ruddle, F.H. 1981. Integration and stable germ line transmission of genes injected into mouse pronuclei. Science. 214:1244-1246.

16. Lindner, V., Fingerle, J., and Reidy, M.A. 1993. Mouse model of arterial injury. Circ. Res. 73:792-796.

17. Groschel Stewart, U., Schreiber, J., and Mahlmeister, C. 1976. Production of specific antibodies to contractile proteins, and their use in immunofluorescence microscopy. I. Antibodies to smooth and striated chicken muscle myosins. Histochemistry. 46:229-236.

18. Shanahan, C.M., Cary, N.R., Metcalfe, J.C., and Weissberg, P.L. 1994. High expression of genes for calcification-regulating proteins in human atherosclerotic plaques. J. Clin. Invest. 93:2393-2402.

19. McHugh, K.M., and Lessard, J.L. 1988. The nucleotide sequence of a rat vascular smooth muscle alpha-actin cDNA. Nucl. Acids Res. 16:4167.

20. Hautmann, M.B., Adam, P.J., and Owens, G.K. 1999. Similarities and differences in smooth muscle alpha-actin induction by TGF-beta in smooth muscle versus non-smooth muscle cells. Arterioscler. Thromb. Vasc. Biol. 19:2049-2058.

21. Geisterfer, A.A., Peach, M.J., and Owens, G.K. 1988. Angiotensin II induces hypertrophy, not hyperplasia, of cultured rat aortic smooth muscle cells. Circ. Res. 62:749-756.

22. Dignam, J.D., Lebovitz, R.M., and Roeder, R.G. 1983. Accurate transcription initiation by RNA polymerase II in a soluble extract from isolated mammalian nuclei. Nucl. Acids Res. 11:1475-1489.

23. Madsen, C.S., Hershey, J.C., Hautmann, M.B., White, S.L., and Owens, G.K. 1997. Expression of the smooth muscle myosin heavy chain gene is regulated by a negative-acting GC-rich element located between two positive-acting serum response factor-binding elements. J. Biol. Chem. 272:6332-6340.

24. Andersen, H., Maeng, M., Thorwest, M., and Falk, E. 1996. Remodeling rather than neointimal formation explains luminal narrowing after deep vessel wall injury: insights from a porcine coronary (re)stenosis model. Circulation. 93:1716-1724.

25. Shi, Y., Pieniek, M., O’Brien, J., Mannion, J., and Zalewski, A. 1996. Adventitial remodeling after coronary arterial injury. Circulation. 93:340-348.

26. Ross, R. 1999. Atherosclerosis: an inflammatory disease. N. Engl. J. Med. 340:115-126.

27. Kuo, M.H., and Allis, C.D. 1998. Roles of histone acetyltransferases and deacetylases in gene regulation. Bioessays. 20:615-626.

28. Watanabe, N., et al. 1999. BTEB2, a Kruppel-like transcription factor, regulates expression of the $\mathrm{SMemb} /$ nonmuscle myosin heavy chain $\mathrm{B}$ (SMemb/NMHC-B) gene. Circ. Res. 85:182-191.

29. Hata, Y., Duh, E., Zhang, K., Robinson, G.S., and Aiello, L.P. 1998. Transcription factors Sp1 and Sp3 alter vascular endothelial growth factor receptor expression through a novel recognition sequence. J. Biol. Chem. 273:19294-19303

30. Rafty, L.A., and Khachigian, L.M. 1998. Zinc finger transcription factors mediate high constitutive platelet-derived growth factor-B expression in smooth muscle cells derived from aortae of newborn rats. J. Biol. Chem. 273:5758-5764.

31. Silverman, E.S., Khachigian, L.M., Lindner, V., Williams, A.J., and Collins, T. 1997. Inducible PDGF A-chain transcription in smooth muscle cells is mediated by Egr-1 displacement of Sp1 and Sp3. Am. J. Physiol. 273:H1415-H1426.

32. Owens, G.K., Vernon, S.M., and Madsen, C.S. 1996. Molecular regulation of smooth muscle cell differentiation. J. Hypertens. 14:S44-S64.

33. Reidy, M.A., Clowes, A.W., and Schwartz, S.M. 1983. Endothelial regeneration. V. Inhibition of endothelial regrowth in arteries of rat and rabbit. Lab. Invest. 49:569-575.

34. Crowley, S.T., Ray, C.J., Nawaz, D., Majack, R.A., and Horwitz, L.D. 1995. Multiple growth factors are released from mechanically injured vascular smooth muscle cells. Am. J. Physiol. 269:H1641-H1647.

35. Rutherford, C., et al. 1997. Substantial inhibition of neo-intimal response to balloon injury in the rat carotid artery using a combination of antibodies to platelet-derived growth factor-BB and basic fibroblast growth factor. Atherosclerosis. 130:45-51. 